\title{
Ovarian endometrioma infiltrating neutrophils orchestrate immunosuppressive microenvironment
}

\author{
Hua $\mathrm{Xu}^{1,2+}$, Jing Zhao ${ }^{1 \dagger}$, Jiaqi Lu, ${ }^{1,3^{*}}$ and Xiaoxi Sun ${ }^{1,2,4^{*}}$
}

\begin{abstract}
Background: Ovarian endometrioma (EM) lesions not only have overwhelmed the amount of infiltrating immune cells but also display immunosuppressive phenotype. The close relationship between neutrophils and the pathogenesis of endometriosis has been demonstrated. The present study aims to elucidate whether or not neutrophils are involved in the regulation of immunosuppressive microenvironment in ovarian endometrioma.

Methods: Immunochemistry (IHC) and flow cytometry analysis (FACS) were conducted to measure CD66b expression in ovarian endometrioma samples from EM patients. The correlation between percentage of CD66b and PD1 + CD8+, TIM3 + CD8+, CTLA4 + CD8+, IFN- + + CD8+ of CD45+ cells were analyzed. Neutrophil survival and PDL1 expression were determined under the stimulations of ovarian endometrioma conditional supernatants (OECS). Finally, CD8+ T cell's proliferation and IFN- $\gamma$ expression were detected under co-cultured with OECS cultured neutrophils stimulated with the a-CD3/a-CD28 antibody.
\end{abstract}

Results: $I H C$ and FACS results revealed correlation between the counts of neutrophils and the severity of ovarian endometrioma. The percentage of CD66b + cells was positively correlated with PD1 + CD8+, TIM3 + CD8+ and CTLA4 + CD8+ of CD45+ cells in ovarian endometrioma. OECS promoted neutrophils' survival and enhanced PD-L1 expression. OECS cultured neutrophils inhibited proliferation and activity of autologous $T$ cells.

Conclusions: Neutrophils play a crucial role in the progression of ovarian endometrioma by orchestrated the immunosuppressive microenvironment under the PD-1/PD-L1 axis.

Keywords: Ovarian endometrioma, Neutrophils, PD-L1, Lymphocytes, Immunosuppression

\section{Introduction}

Endometriosis (EM) is defined as a chronic inflammatory disease characterized by ectopic presence of endometrial glands and stroma predominantly in the pelvic compartment, and is a common disorder affects women of reproductive period with pelvic pain and reduced fertility [1]. Three types of EM have been characterized: peritoneal superficial endometriosis (SUP), ovarian endometrioma

\footnotetext{
*Correspondence: lujiaqidoc@hotmail.com; xiaoxi_sun@aliyun.com

${ }^{\dagger}$ Hua Xu and Jing Zhao contributed equally to this work.

'Obstetrics and Gynecology Hospital, Fudan University, Shanghai 200011, China

Full list of author information is available at the end of the article
}

(OMA), and deeply infiltrating endometriosis(DIE) [2, 3]. The involvement of various immune cells such as $\mathrm{T}$ and $\mathrm{B}$ lymphocytes, natural killer cells, macrophages, and dentritic cells have been demonstrated in peritoneal inflammation and endometriosis lesion establishment $[4,5]$. Endometriosis lesions not only have overwhelmed amount of infiltrating immune cells, but these cells often exhibit immunosuppressive phenotype, suggesting that defects of immune surveillance generated by suboptimal immune response that lead to the initiation and development of endometriosis [6].

Recently, the close relationship between neutrophils and the pathogenesis of endometriosis has been demonstrated. 
For instance, accumulation of neutrophils has been found in the peritoneal fluid of ovarian endometriosis [7, 8]. Neutrophil extracellular traps (NETs) produced by activated neutrophils are present in the peritoneal fluid of endometriosis patients [9]. Neutrophil infiltrating the human endometrium express VEGF and regulate cyclical endometrial vascular proliferation [10]. Endometriosis is associated with increased percentage of peritoneal neutrophils and that the levels of human neutrophil peptides (HNP 1-3) strongly correlated with the percentage of neutrophils [8]. Depletion neutrophils significantly reduced the severity of endometriotic lesions [11]. However, the role of neutrophil in the immunosuppressive microenvironment of ovarian endometrioma remains unknown.

Neutrophils are highly plasticity implying that these cells may differentiate into discrete subsets under pathological conditions [12]. For instance, neutrophils display not only protumor but also anti-tumor properties depending on tumor growth factor- $\beta$ (TGF- $\beta$ ) within the tumor microenvironment [13]. Neutrophils adopted anti-inflammatory phenotype during Mycobacterium tuberculosis infection [14]. Furthermore, neutrophils contribute to orientation of adaptive immune responses by suppression of $\mathrm{T}$ cell proliferation and activity under the control of interleukin-10 (IL-10), arginase 1, and reactive oxygen species [15]. In the gastric cancer, tumor activated neutrophils foster immune suppression may be a result of PD1/PD-L1 axis [16]. Notably, the phenotype and function relevance of neutrophils in the immunosuppressive microenvironment of ovarian endometriosis remain unknown.

Herein, we found the association between the severity of endometriosis and the counts of infiltrating neutrophils. Moreover, we demonstrated that ovarian endometrioma prolonged neutrophil lifespan and induced programmed death-ligand 1 (PD-L1) expression on neutrophils. In turn, these neutrophils suppress $T$ cell's proliferation and activation, indicating PD-L1 expression neutrophils may contribute to the suppression of adaptive immune response during the progression of ovarian endometrioma.

\section{Materials and methods}

\section{Ovarian endometrioma tissue and peripheral blood} collection

Endometriotic tissue specimens were obtained from 56 women with ovarian endometrioma who underwent laparoscopic surgery at the Department of Obstetrics and Gynecology, Obstetrics and Gynecology Hospital, Fudan University, from March 2016 to Jun2016. Cohort 1 ( $n=$ $35)$ were subjected to immunohistochemical analysis. Cohort $2(n=21)$ were subjected to flow cytometry analysis. Their major clinical and surgical features are presented in Table 1. The clinical characteristics are balance between two cohorts. All ovarian endometrioma subjects were histologically proven endometrioma. During surgery, endometriosis was staged and scored (total, implant, and adhesion scores) according to the revised American Fertility Society (rAFS) classification [17]. Women with isolated ovarian endometrioma corresponded to women with ovarian endometrioma associated peritoneal endometriosis with adhesions, exclusive of deeply infiltrative endometriosis [18]. Furthermore, women with infectious diseases such as hepatitis B or C virus, or human immunodeficiency virus and women with malignancies or autoimmune diseases were excluded in this study. None of the included women were pregnant.

Whole blood collected from 10 health adult female donors (aged 20-50 years) was used to isolate neutrophils and peripheral blood mononuclear cells (PBMCs).Neutrophils and PBMC prepared using PolymorphPrep (Axis-Shield) and LymphoPrep (Axis-Shield) following the manufacturer's protocol. This study was approved by the Ethics Committee of the Affiliated Obstetrics and Gynecology Hospital of Fudan University. Written informed consent was obtained from each participant in accordance with the approved guidelines.

\section{Immunohistochemistry}

Paraffin sections $(5 \mu \mathrm{m})$ of ovarian endometrioma tissue were deparaffinized in xylene, rehydrated in graded

Table 1 Clinical characteristics of 56 cases with ovarian endometriosis

\begin{tabular}{llll}
\hline Characteristic & Cohort 1(n=35) & Cohort 2 $(n=21)$ & $32(23-44)$ \\
\hline Age (yr) & $31(23-46)$ & & 0.461 \\
Name of operation & & $5(23.81 \%)$ \\
$\quad$ Laparoscopic right ovarian cystectomy & $9(25.71 \%)$ & $7(33.33 \%)$ \\
$\quad$ Laparoscopic left ovarian cystectomy & $11(31.43 \%)$ & $9(42.86 \%)$ & $41(28-130)$ \\
$\quad$ Laparoscopic cystectomy of both ovaries & $15(42.86 \%)$ & $7(1.5-11)$ & 0.983 \\
Endometriosis score by rASRM criteria & $39(22-132)$ & $10(47.62 \%)$ & 0.182 \\
The largest diameter of endometrioma (cm) & $6(3-10)$ & & 0.415 \\
Dysmenorrhea & $21(60 \%)$ &
\end{tabular}

Values are presented as mean (range) or number (\%) rASRM revised American Society for Reproductive Medicine 
ethanol, and subjected to antigen retrieval using sodium citrate buffer (10 mM, pH 6.0). The sections were blocked with $\mathrm{H}_{2} \mathrm{O}_{2}$ and blocking buffer (2.5\% bovine serum albumin (BSA) in phosphate buffer saline (PBS). The sections were then incubated with mouse anti-human CD66b (1: 600, Biolegend). Horseradish peroxidase conjugated secondary antibodies (Vector Laboratories) were applied for $30 \mathrm{~min}$ and visualized with 3'3-diaminobenzidine (Vector Laboratories). Staining with isotype antibody was used as negative control. Images were taken on the Nikon Eclipse 80i microscope (Nikon, Japan). The neutrophil was determined as number of cells/high power field (HPF) using an algorithm developed for National Institutes of Health (NIH) software ImageJ.

\section{Preparation of OECS and supernatant-conditioned neutrophils}

Ovarian endometrioma tissue culture supernatants (OECS) were prepared by plating ovarian endometrioma tissues in $1 \mathrm{~mL}$ RPMI-1640 medium for $24 \mathrm{~h}$. The supernatant was then centrifuged and harvested. Four respect ovarian endometrioma specimens were prepared for OECS. To generate supernatant-conditioned neutrophils, neutrophils were cultured with 50\% OECS for $12 \mathrm{~h}$, then washed with RPMI-1640 medium for three times. Neutrophils cultured with RPMI-1640 medium were used as controls.

\section{Neutrophils survival assay}

Neutrophils from healthy donors were stimulated with $30 \%$ or $60 \%$ OECS for $16 \mathrm{~h}$, and then were harvested. Neutrophils survival was quantified using Annexin V Apoptosis Detection Kit (BD biosciences) according to the manufacturer's instructions.

\section{Measurement of CD8+ $\mathrm{T}$ cells proliferation with CFSE}

Measurement of $\mathrm{CD}^{+} \mathrm{T}$ cells proliferation with5-(and 6)-Carboxyfluorescein diacetate succinimidyl ester (CFSE) was applied as previously described [19]. PBMC were resuspended at $1 \times 10^{7}$ cells $/ \mathrm{mL}$ in PBS containing $5 \mu \mathrm{M}$ CFSE (Biolegend). After $20 \mathrm{~min}$ incubation at room temperature, cells were washed twice with culture medium. Then the PBMC were stimulated with antiCD3 Ab $(1 \mu \mathrm{g} / \mathrm{mL})$, anti-CD28 Ab $(1 \mu \mathrm{g} / \mathrm{mL})$ and IL-2 $(100 \mathrm{U} / \mathrm{mL})$ in the presence or absence of OECS conditioned neutrophils for 3 days. Cells were then stained with $\mathrm{CD} 8 \mathrm{mAb}$, and CFSE positive $\mathrm{CD}^{+} \mathrm{T}$ cells were finally analyzed by a flow cytometer (Beckman CytoFLEX) using CytExpert Software.2.7.

\section{Analysis of intracellular IFN- $ү$ production by flow cytometry}

PBMC $\left(2 \times 10^{6}\right.$ cells $\left./ \mathrm{mL}\right)$ were stimulated with immobilized anti-CD3/anti-CD28 (each at $1 \mu \mathrm{g} / \mathrm{mL}$ ) and IL-2
$(100 \mathrm{U} / \mathrm{mL})$ in the absence or presence of OECS conditioned neutrophils. After $24 \mathrm{~h}, 1 \times$ protein transport inhibitor cocktail (Invitrogen) was added for the last $6 \mathrm{~h}$. Following stimulation, cells were harvested, washed in PBS and incubated for $30 \mathrm{~min}$ at $4{ }^{\circ} \mathrm{C}$ in the darkness with the anti-CD8 $\mathrm{mAb}$ (Biolegend). Cells were then washed once in PBS containing 2\% v/v FCS prior to fixation and permeabilization with $500 \mu \mathrm{L}$ Cytofix/Cytoperm solution (Becton Dickinson) for $10 \mathrm{~min}$ at room temperature in darkness. Cells were washed once again and incubated with blocking buffer for $20 \mathrm{~min}$ at $4{ }^{\circ} \mathrm{C}$ in the darkness to prevent unspecific binding of the anticytokine monoclonal antibody. After another washing step, $5 \mu \mathrm{L}$ anti-IFN- $\gamma$-FITC (Biolegend) were added and cells were incubated for $30 \mathrm{~min}$ at $4{ }^{\circ} \mathrm{C}$ in the darkness followed by a final washing step prior to measurement. Measurement was done using flow cytometer (Beckman CytoFLEX) using CytExpert Software.2.7.

\section{Statistical analysis}

Student's t tests were used to compare differences in the means of the continuous variable between the groups of patients with two cohorts of endometriosis. The relationship between the endometriosis score and each of parameters were evaluated using the nonparametric Spearman's rank correlation test. All statistical analyses were performed using Prism (Graphpad). A P-value < 0.05 was considered statistically significant.

\section{Results}

\section{Patient characteristics}

Fifty-six ovarian endometrioma affected women were recruited for this study. Their major clinical and surgical features are presented in Table 1.Ovarian endometrioma patients were distributed among rAFS stages III and IV as follows: 24 (42.9\%) and 32 (57.1\%), respectively. Mean total, implants, and adhesion rAFS scores were $56.1 \pm$ $31.4,27.3 \pm 8.7,28.6 \pm 27.4$, respectively.

\section{Neutrophils accumulated in the ovarian endometrioma}

We detected neutrophil infiltration in ovarian endometrioma specimens with CD66b staining in immunohistochemistry. The results showed that the amount of infiltrating neutrophils was positively correlated with the severity scores of patients with ovarian endometrioma $(r=0.465, P=0.005)$ (Fig. 1a and b). Consistently, the association between the severity of ovarian endometrioma and the percentage of $\mathrm{CD} 66 \mathrm{~b}^{+}$of $\mathrm{CD} 45^{+}$cells were confirmed ( $r=0.438, P=0.047)$ (Fig. 1c).

\section{Neutrophils infiltrating ovarian endometrioma were defined with immunosuppressive phenotype}

Next, significant positive correlations were found between the levels of $\mathrm{CD} 6 \mathrm{~b}^{+}$and $\mathrm{PD} 1^{+}, \mathrm{Tim} 3^{+}$, and 

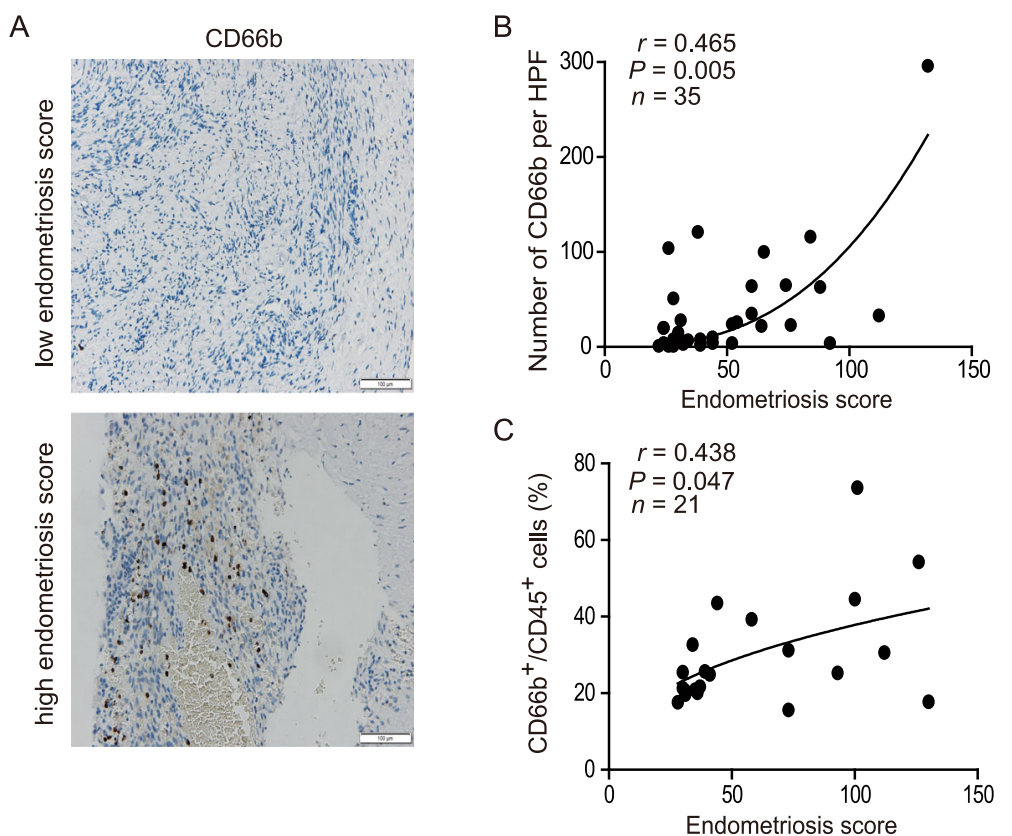

Fig. 1 Ovarian endometriosis infiltrating neutrophils were correlated with endometriosis scores. a Representative image showing neutrophils infiltrating in ovarian endometriosis specimens. Bar $=100 \mu \mathrm{M}$. b The correlation between the endometriosis scores and the number of CD66b per high power field (HPF) in ovarian endometriosis specimens detected by $\mathrm{IHC}(n=35)$. c The correlation between the endometriosis scores and the percentage of $\mathrm{CD}_{6} 6 \mathrm{~b}^{+}$of $\mathrm{CD} 45^{+}$cells in ovarian endometriosis specimens detected by FACS $(n=21)$

CTLA4 $^{+}$expression on $\mathrm{CD}^{+} \mathrm{T}$ cells (Fig. 2a-c). However, there was no correlation between the levels of $\mathrm{CD} 6 \mathrm{~b}^{+}$and IFN- $\gamma^{+}$expression on $\mathrm{CD}^{+} \mathrm{T}$ cells (Fig. 2d).

\section{Ovarian endometrioma environments contribute to neutrophil survival and maintain neutrophil activated immunosuppressive phenotype}

We hypothesized that ovarian endometrioma environment sustained the survival of neutrophils. To test this hypothesis, we assessed the survival of neutrophils after exposure to OECS, and the results showed that neutrophils exposed to OECS exhibited a delayed onset of apoptosis in a dose-dependent manner when compared to those exposed to medium by annexin V (Fig. 3a). Given the immunosuppressive microenvironment in the ovarian endometrioma, we hypothesized that the endometrioma microenvironment itself might play an important role in this process. PBMC were co-cultured with OECS-conditioned neutrophils. OECS-conditioned neutrophils significantly suppressed $\mathrm{T}$ cell proliferation and less IFN- $\gamma$ production (Fig. 3b, C).

\section{PD-L1 expression in neutrophils are found in ovarian endometrioma}

Meanwhile, we also hypothesized that OECS environments contribute to the immunosuppressive phenotype of neutrophils. Consistent with our hypothesis, compared with medium-conditioned neutrophils, OECS significantly up-regulated PD-L1 expression (Fig. 4a). Consistently PD-L1 expression levels were positively correlated with the percentage of $\mathrm{CD} 6 \mathrm{~b}^{+}$cells in ovarian endometrioma specimens (Fig. 4b).

\section{Discussion}

Endometriosis is a chronic inflammatory disease driven by dysregulated immune system, which facilitates endometrial cells implantation, proliferation, and promotes molecular abnormalities within the eutopic endometrium [20]. Peritoneal neutrophils and macrophagedriven inflammatory networks enhance endometriotic cell survival, cell growth, cell invasion, and angiogenesis $[11,21]$. Suppression of cytotoxicity of NK cells and imbalance of $\mathrm{T}$ cell subsets has limited capability to eliminate endometrial cells in the peritoneal cavity [22, 23]. In contrast, studies that focus on endometriosis lesion infiltrating immune cells are very limited. Here, we found statistically significant correlations between the ovarian endometrioma infiltrating neutrophils and the severity of endometriosis. Ovarian endometrioma microenvironment induced PD-L1 expression in neutrophils, which acquired the ability to suppress CD8 T cell proliferation and activation.

Neutrophils are the first line to defense against microbial infections and play a pivotal role in inflammatory disease [24]. In the peripheral fluid of endometriosis, 


$$
\text { A }
$$

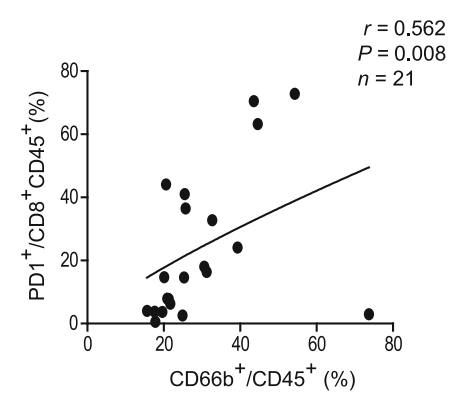

C

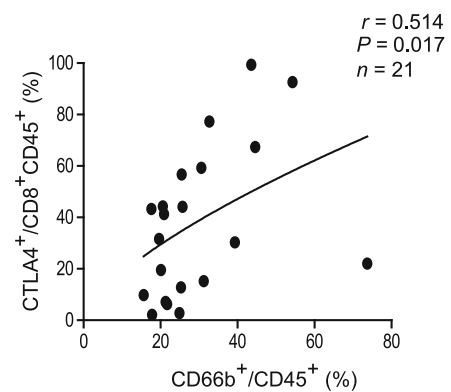

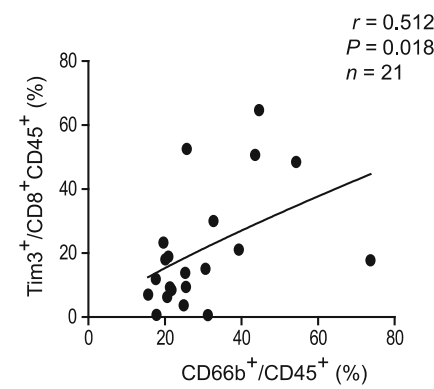

D

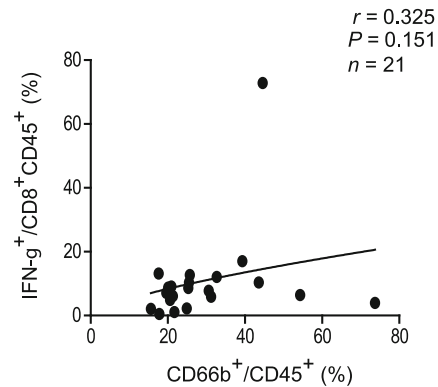

Fig. 2 Ovarian endometriosis infiltrating neutrophils were correlated with the number of immune checkpoints positive CD8 T cells. a-d The correlation between the percentage of $\mathrm{CD}_{6} 6 \mathrm{~b}^{+}$of $\mathrm{CD} 45^{+}$cells and the percentage of PD $1^{+}$of $\mathrm{CD} 8^{+} \mathrm{CD} 45^{+}$lymphocytes (A), the percentage of $\mathrm{Tim}^{+}$of $\mathrm{CD} 8^{+} \mathrm{CD} 45^{+}$lymphocytes (b), the percentage of $\mathrm{CTLA} 4^{+}$of $\mathrm{CD} 8^{+} \mathrm{CD} 45^{+}$lymphocytes (C), the percentage of IFN- $\gamma^{+}$of $\mathrm{CD} 8^{+} \mathrm{CD} 45^{+}$ lymphocytes (d)

increased amounts of neutrophils has been observed, especially in the advanced stages $[8,25]$. In the blood of endometriosis, increased neutrophil-to-lymphocyte ratio has been found and proposed as a potential measure of disease severity $[22,26]$. Moreover, a substantial infiltration of neutrophils into the peritoneal cavity occurred during early initiation of uterine tissue transfer mouse model of endometriosis [27]. Early depletion of neutrophils by anti-granulocyte receptor (Gr-1) antibody relieved the formation of endometriotic lesion, which suggests that neutrophils play an important role in the initiation and progression of endometriosis. In the present study, we found that amounts of lesion infiltrating neutrophils were correlated with the severity of the ovarian endometrioma.

CXC motif ligand 8 (CXCL8/IL8), the most potent neutrophil chemoattractant, contributes to recruit neutrophils by binding to the receptors CXCR1 and CXCR2. Other chemokines such as CXCL1, CXCL2, CXCL3, and G-CSF can also attract neutrophils [28]. Consistently, patients with endometriosis displayed a significant higher production of CXCL1, CXCL2, and CXCL8 in the peritoneal fluid as compared to those from controls [29-31]. Secretion of CXCL8 is significantly higher in the eutopic endometrial stromal cells from women with endometriosis than that in the control [32]. The elevated concentration of neutrophil chemoattractants may be responsible for the recruitment of neutrophils in the peritoneal fluid and endometriosis lesion.

Neutrophils not only promote endometriosis growth by secreting a variety of cytokines and chemokines, but also enhance endometriosis angiogenesis, migration and invasion by providing enzymes that deregulate the extracellular matrix (ECM). Neutrophils are able to release numerous mediators, including proinflammatory (e.g., IL-1 $\alpha / \beta$, IL-6, IL-8, IL-9, IL-16), angiogenic (e.g., vascular endothelial growth factor (VEGF) and anti-inflammatory mediators (e.g. tumor growth factor (TGF- $\beta$ ), IL-4, IL-10) [15].Within the surrounding peritoneal fluid, numerous cytokines (e.g., TNF- $\alpha$, IL-10, and TGF- $\beta$ ), chemokines (e.g., IL-8), angiogenic growth factors (e.g., VEGF) are present [33]. Neutrophils in the peritoneal fluid of endometriosis increase the production of VEGF in the presence of IL-6, TNF- $\alpha$, LPS, and estrogen [34]. Matrix metalloproteinase (MMP9) expression increased in the endometrium from women with endometriosis [35]. Furthermore, higher neutrophil extracellular traps (NETs) levels in the peritoneal fluid of patients with endometriosis has been found, which seem to be 


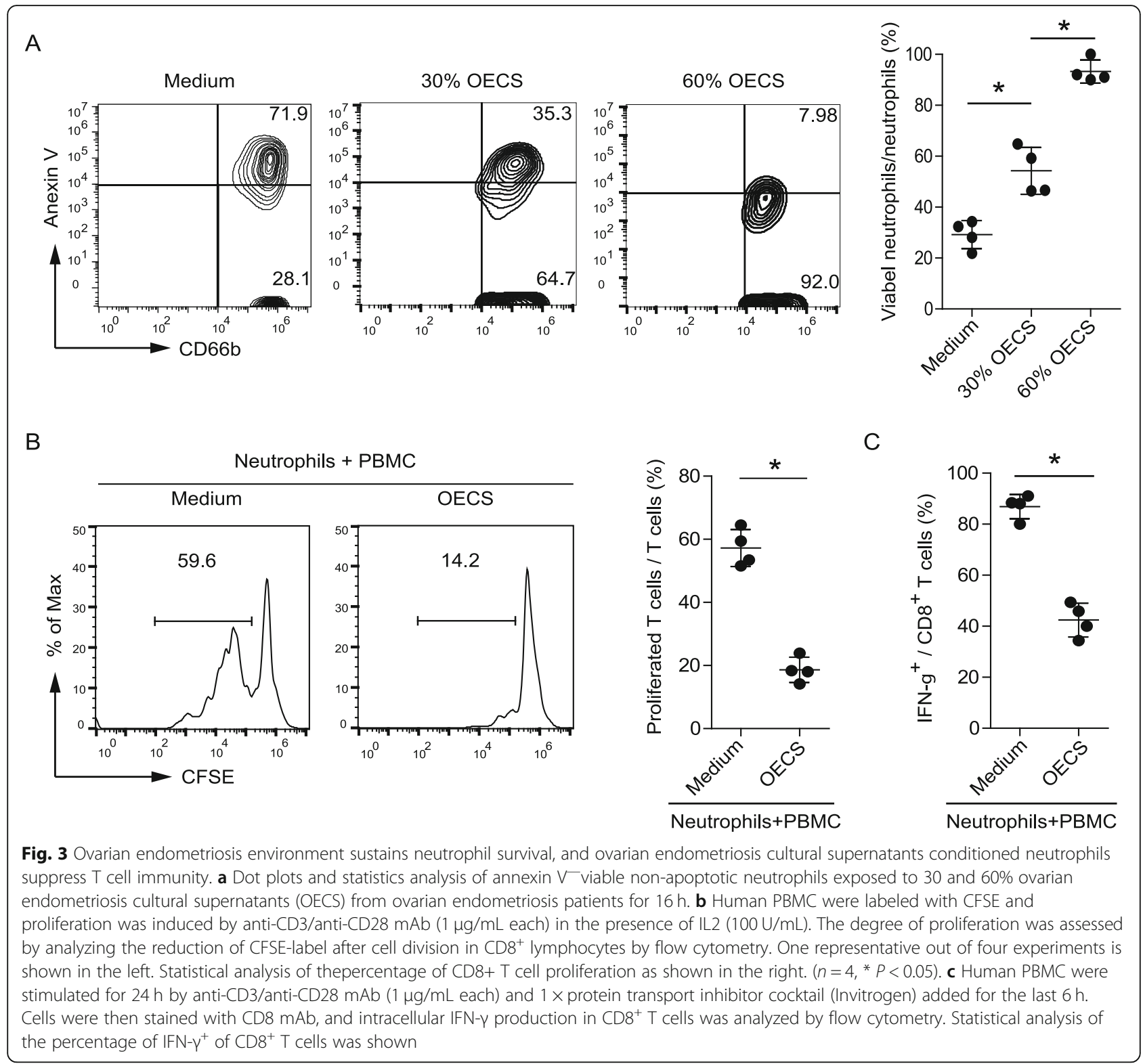

implicated in its pathogenesis [9]. Neutrophils also contribute to immune suppression to enhance endometriosis growth. This together with the fact that IFN- $\gamma$ stimulated PD-L1 expression in neutrophils and acquire the capacity to suppress $\mathrm{T}$ cell proliferation [36]. Although neutrophils have already been described in patients with endometriosis, the alterations of functions of neutrophils in the endometriosis microenvironment remain to be elucidated. Here, we found that increased PD-L1 expression in neutrophils in the presence of ovarian endometrioma supernatants, which inhibit CD8 $\mathrm{T}$ cell proliferation and activation.

The primary limitation of our study included only women who underwent surgery for ovarian endometrioma and were diagnosed with moderate to severe endometriosis. There is lack of adjacent healthy ovarian tissues applied for preparation of culture supernatants. In summary, this study demonstrates that neutrophils are correlated with the severity of ovarian endometrioma and inhibit CD8 $\mathrm{T}$ cell immunity under PD-L1 dependent manner, although factors that mediate neutrophil PD-L1 expression required further investigation. These findings add to our current understanding of disease development and inform new strategies for the prevention and management of ovarian endometrioma.

\section{Conclusions}

In conclusion, our results indicated that the amount of neutrophils was associated with the severity of ovarian endometriosis. The ovarian endometriosis associated 

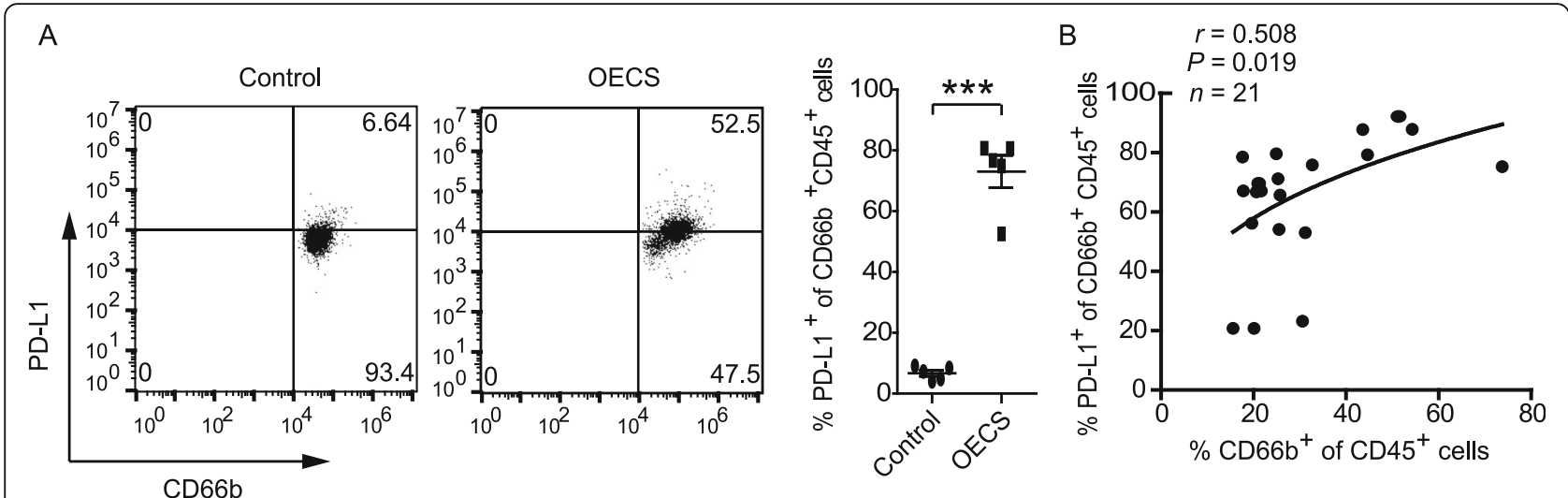

Fig. 4 Ovarian endometriosis environment sustains PD-L1 expression on neutrophils. a Dot plots and statistics analysis of PD-L $1^{+}$expression on neutrophils exposed to $60 \%$ ovarian endometriosis cultural supernatants (OECS) from ovarian endometriosis patients for $12 \mathrm{~h}$. $\left(n=5,{ }^{* * *} P<0.001\right)$. b The correlation between the percentage of $\mathrm{CD}_{6} 6 \mathrm{~b}^{+}$of $\mathrm{CD}_{4} 5^{+}$cells and the percentage of $\mathrm{PD}-\mathrm{L}^{+}{ }^{+}$of $\mathrm{CD} 66 \mathrm{~b}^{+} \mathrm{CD} 45^{+}$cells

neutrophils inhibits the activities of CD8+ $\mathrm{T}$ cells in vitro, and may play a role in the development of immunosuppressive microenvironment by PD-L1 expression in vivo. Moreover, these conclusions should be validated by more stringent controls.

\section{Abbreviations}

BSA: Bovine serum albumin; CFSE: 5-(and 6)-carboxyfluorescein diacetate succinimidyl ester; CXCL8: cxc motif ligand 8; DIE: Deeply infiltrating endometriosis; EM: Endometrioma; FACS: Flow cytometry analysis; HNP: Human neutrophil peptides; Gr: Granulocyte receptor; HPF: High power field; IHC: Immunochemistry; IL: Interleukin; MMP: Matrix metalloproteinase; NETs: Neutrophil extracellular traps; NIH: National Institutes of Health; OECS: Ovarian endometrioma conditional supernatants; OMA: Ovarian endometrioma; PBMC: Peripheral blood mononuclear cells; PBS: Phosphate buffer saline; PD-L1: Programmed death ligand 1; Rafs: Revised American Fertility Society; SUP: Peritoneal superficial endometriosis; TGF- $\beta$ : Tumor growth factor- $\beta$; VEGF: Vascular endothelial growth factor

\section{Acknowledgements}

None.

\section{Authors' contributions}

L: Project development, Data analysis, Manuscript writing. XH: Data collection, Data analysis. ZJ: Data collection and analysis. SXX: Project supervised and funding support. All authors read and approved the final manuscript.

\section{Funding}

This study was funded by grants from Natural Science Foundation of Xinjiang Uygur Autonomous Region (2019D01C088).

\section{Availability of data and materials}

All publicly data generated or analyzed during this study are included in this published article. The data used to support the findings of this study are available from the corresponding author upon request.

\section{Ethics approval and consent to participate}

This study was approved by the Medical Institutional Ethics Committee of Gynecology and Obstetrics Hospital of Fudan University. Informed consent was obtained from all the enrolled patients who underwent surgery.

\section{Consent for publication}

Not applicable.

\section{Competing interests}

The authors declare no conflict of interest.

\section{Author details}

${ }^{1}$ Obstetrics and Gynecology Hospital, Fudan University, Shanghai 200011 China. ${ }^{2}$ Shanghai Ji Ai Genetics and IVF Institute, Obstetrics and Gynecology Hospital, Fudan University, Shanghai 200011, China. ${ }^{3}$ Department of Gynecology, Second People's Hospital in Kashgar, Xinjiang Uyhur Autonomous Region. No.1, Health Road, Kashgar 844000, Xinjiang, China. ${ }^{4}$ Key laboratory of Female Reproductive endocrine Related Diseases, Obstetrics and Gynecology Hospital, Fudan University, Shanghai 200011, China.

Received: 12 October 2019 Accepted: 31 March 2020

Published online: 25 April 2020

\section{References}

1. Giudice LC. Clinical practice. Endometriosis. N Engl J Med. 2010;362(25): 2389-98.

2. Fauconnier A, Chapron C. Endometriosis and pelvic pain: epidemiological evidence of the relationship and implications. Hum Reprod Update. 2005; 11(6):595-606.

3. de Ziegler D, Borghese B, Chapron C. Endometriosis and infertility: pathophysiology and management. Lancet. 2010;376(9742):730-8.

4. Herington JL, Bruner-Tran KL, Lucas JA, Osteen KG. Immune interactions in endometriosis. Expert Rev Clin Immunol. 2011;7(5):611-26.

5. Osuga Y, Koga K, Hirota Y, Hirata T, Yoshino O, Taketani Y. Lymphocytes in endometriosis. Am J Reprod Immunol. 2011;65(1):1-10.

6. Ahn SH, Khalaj K, Young SL, Lessey BA, Koti M, Tayade C. Immuneinflammation gene signatures in endometriosis patients. Fertil Steril. 2016; 106(6):1420-31 e1427.

7. Takamura M, Osuga Y, Izumi G, et al. Interleukin-17A is present in neutrophils in endometrioma and stimulates the secretion of growthregulated oncogene-alpha (Gro-alpha) from endometrioma stromal cells. Fertility and sterility. 2012;98(5):1218 1224 e1211-1212.

8. Milewski L, Dziunycz P, Barcz E, et al. Increased levels of human neutrophil peptides 1, 2, and 3 in peritoneal fluid of patients with endometriosis: association with neutrophils, T cells and IL-8. J Reprod Immunol. 2011;91(12):64-70.

9. Berkes E, Oehmke F, Tinneberg HR, Preissner KT, Saffarzadeh M. Association of neutrophil extracellular traps with endometriosis-related chronic inflammation. Eur J Obstet Gynecol Reprod Biol. 2014;183:193-200.

10. Mueller MD, Lebovic DI, Garrett E, Taylor RN. Neutrophils infiltrating the endometrium express vascular endothelial growth factor: potential role in endometrial angiogenesis. Fertil Steril. 2000;74(1):107-12.

11. Takamura M, Koga K, Izumi G, et al. Neutrophil depletion reduces endometriotic lesion formation in mice. Am J Reprod Immunol. 2016;76(3): 193-8.

12. Scapini $P$, Cassatella MA. Social networking of human neutrophils within the immune system. Blood. 2014;124(5):710-9. 
13. Fridlender ZG, Sun J, Kim S, et al. Polarization of tumor-associated neutrophil phenotype by TGF-beta: "N1" versus "N2" TAN. Cancer Cell. 2009; 16(3):183-94.

14. Zhang X, Majlessi L, Deriaud E, Leclerc C, Lo-Man R. Coactivation of Syk kinase and MyD88 adaptor protein pathways by bacteria promotes regulatory properties of neutrophils. Immunity. 2009;31(5):761-71.

15. Mantovani A, Cassatella MA, Costantini C, Jaillon S. Neutrophils in the activation and regulation of innate and adaptive immunity. Nat Rev Immunol. 2011;11(8):519-31.

16. Wang $\Pi$, Zhao $Y L$, Peng $L S$, et al. Tumour-activated neutrophils in gastric cancer foster immune suppression and disease progression through GMCSF-PD-L1 pathway. Gut. 2017;66(11):1900-11.

17. Revised American Fertility Society classification of endometriosis: 1985. Fert steril. 1985;43(3):351-352.

18. Santulli P, Borghese B, Chouzenoux S, et al. Interleukin-19 and interleukin-22 serum levels are decreased in patients with ovarian endometrioma. Fertil Steril. 2013;99(1):219-26.

19. Naundorf S, Schroder M, Hoflich C, Suman N, Volk HD, Grutz G. IL-10 interferes directly with TCR-induced IFN-gamma but not IL-17 production in memory T cells. Eur J Immunol. 2009;39(4):1066-77.

20. Bulun SE. Endometriosis. N Engl J Med. 2009;360(3):268-79.

21. Beste MT, Pfaffle-Doyle N, Prentice EA, et al. Molecular network analysis of endometriosis reveals a role for c-Jun-regulated macrophage activation. Sci Transl Med. 2014;6(222):222ra216.

22. Izumi G, Koga $\mathrm{K}$, Takamura $\mathrm{M}$, et al. Involvement of immune cells in the pathogenesis of endometriosis. J Obstet Gynaecol Res. 2018:44(2):191-8.

23. Wu MY, Yang JH, Chao KH, Hwang JL, Yang YS, Ho HN. Increase in the expression of killer cell inhibitory receptors on peritoneal natural killer cells in women with endometriosis. Fertil Steril. 2000;74(6):1187-91.

24. Kolaczkowska E, Kubes P. Neutrophil recruitment and function in health and inflammation. Nat Rev Immunol. 2013;13(3):159-75.

25. Tariverdian N, Siedentopf F, Rucke M, et al. Intraperitoneal immune cell status in infertile women with and without endometriosis. J Reprod Immunol. 2009;80(1-2):80-90

26. Cho $\mathrm{S}, \mathrm{Cho} \mathrm{H}, \mathrm{Nam} \mathrm{A}$, et al. Neutrophil-to-lymphocyte ratio as an adjunct to CA-125 for the diagnosis of endometriosis. Fertil Steril. 2008;90(6):2073-9.

27. Burns KA, Thomas SY, Hamilton KJ, Young SL, Cook DN, Korach KS. Early endometriosis in females is directed by immune-mediated estrogen receptor alpha and IL-6 cross-talk. Endocrinology. 2018;159(1):103-18.

28. Lazennec G, Richmond A. Chemokines and chemokine receptors: new insights into cancer-related inflammation. Trends Mol Med. 2010;16(3):133-44.

29. Marianna S, Alessia P, Susan C, et al. Metabolomic profiling and biochemical evaluation of the follicular fluid of endometriosis patients. Mol BioSyst. 2017; 13(6):1213-22.

30. Borrelli GM, Kaufmann AM, Abrao MS, Mechsner S. Addition of MCP-1 and MIP-3beta to the IL-8 appraisal in peritoneal fluid enhances the probability of identifying women with endometriosis. J Reprod Immunol. 2015;109:66-73.

31. Chen Y, Wang $K$, Xu Y, et al. Alteration of myeloid-derived suppressor cells, chronic inflammatory cytokines, and Exosomal miRNA contribute to the peritoneal immune disorder of patients with endometriosis. Reprod Sci. 2019;26(8):1130-8. Epub on 2018 Nov 19.

32. Li MQ, Luo XZ, Meng YH, et al. CXCL8 enhances proliferation and growth and reduces apoptosis in endometrial stromal cells in an autocrine manner via a CXCR1-triggered PTEN/AKT signal pathway. Hum Reprod. 2012;27(7): 2107-16.

33. Symons LK, Miller JE, Kay VR, et al. The Immunopathophysiology of endometriosis. Trends Mol Med. 2018;24(9):748-62.

34. Lin YJ, Lai MD, Lei HY, Wing LY. Neutrophils and macrophages promote angiogenesis in the early stage of endometriosis in a mouse model. Endocrinology. 2006;147(3):1278-86

35. Yang M, Jiang C, Chen H, Nian Y, Bai Z, Ha C. The involvement of osteopontin and matrix metalloproteinase- 9 in the migration of endometrial epithelial cells in patients with endometriosis. Reprod Biol Endocrinol. 2015;13:95

36. Bankey PE, Banerjee $\mathrm{S}$, Zucchiatti A, et al. Cytokine induced expression of programmed death ligands in human neutrophils. Immunol Lett. 2010; 129(2):100-7.

\section{Publisher's Note}

Springer Nature remains neutral with regard to jurisdictional claims in published maps and institutional affiliations.

Ready to submit your research? Choose BMC and benefit from:

- fast, convenient online submission

- thorough peer review by experienced researchers in your field

- rapid publication on acceptance

- support for research data, including large and complex data types

- gold Open Access which fosters wider collaboration and increased citations

- maximum visibility for your research: over $100 \mathrm{M}$ website views per year

At BMC, research is always in progress.

Learn more biomedcentral.com/submissions 